\title{
Diagnosis of metastases from testicular germ cell tumours using fine needle aspiration cytology
}

\author{
WILMA J HIGHMAN, * R T D OLIVER
}

From the Departments of *Histopathology and Oncology, St Peter's Hospitals and the Institute of Urology, London

SUMMARY The cytological features of testicular germ cell tumours were established in smears from 15 freshly resected tumours. These features were applied to the fine needle aspiration cytology diagnosis of metastases in 27 patients referred for chemotherapy. There were 16 positive reports in 32 aspirates of which 13 were taken before chemotherapy and three in patients with residual or new masses after chemotherapy. Teratomas and typical seminomas showed certain characteristic morphological features in cytological preparations which when present in fine needle aspiration cytology material enabled tumour types to be diagnosed. Spermatocytic and anaplastic seminoma were not represented in this series. It is unlikely that these could be distinguished from malignant teratoma undifferentiated (MTU) in the fine needle aspiration cytology material. Metastases from carcinomatous areas in MTU and malignant teratoma intermediate (MTI) may not be distinguishable in fine needle aspiration cytology material from metastatic adenocarcinoma or undifferentiated carcinoma from a different primary site. Positive cytological findings are of value to the oncologist in the management of patients with metastases from testicular germ cell tumours. negative cytology does not exclude the presence of viable tumour. The sampling of small foci of viable tumour in large necrotic masses persisting after chemotherapy is a problem for radiologists $\stackrel{0}{2}$ cytologists, and histopathologists. This paper does not advocate the use of fine needle aspiration cytology for the diagnosis of primary testicular tumour.

The management of patients with testicular tumours has undergone a revolution in the past decade. New imaging techniques, the use of tumour markers, and the development of effective chemotherapeutic agents have made metastatic testicular cancer curable in all cases except those with very advanced disease. ${ }^{1}$ Germ cell tumours vary considerably in their growth rate. In tumours of rapid growth, in particular the undifferentiated teratomas, delay in diagnosis adversely affects the prognosis by increasing the risk of metastasis and diminishing the chance of cure by cytotoxic drugs once metastases have developed. ${ }^{2}$ Tissue diagnosis entails laparotomy with its attendant potential for complications and delay in the start of treatment. Fine needle aspiration cytology offers the possibility of rapid diagnosis with minimal trauma. In a series of 27 patients referred to the oncology department of St Peter's Hospitals for chemotherapy of

Accepted for publication 9 June 1987 testicular tumour with metastases, fine needle aspiration cytology was performed to establish or confirm the diagnosis.

Little has been written about the features of testicular germ cell tumours in cytological material. ${ }^{2-4}$ Papers on the use of fine needle aspiration cytology in the diagnosis of metastases have essentially been radiological reports. ${ }^{5-8}$ An initial study was therefore undertaken on smears from fresh resected primary testicular tumours. These were used to define the features of the different types of tumour as they presented in cytological preparations and to apply them to the diagnosis of metastatic tumour in fine needle aspiration cytology specimens. This paper describes the cytological features seen in smears from this group of 15 primary resected tumours of the testis and those in the metastases in 27 patients to assess the reliability and role of fine needle aspiration cytology in the diagnosis of metastatic testicular tumour.

The classification of the tumours used throughout is based on that evolved by the British Testicular Tu- 
mour Panel. ${ }^{910}$ Thus teratoma refers to a tumour that is composed of multiple tissues of all kinds foreign to the normal testis, and showing a greater or lesser degree of cell differentiation. Teratoma differentiated (TD) refers to the most highly differentiated in which no histological malignant areas can be found (mature teratoma in the WHO classification $^{11}$ ). Malignant teratoma intermediate (MTI) refers to tumours containing both differentiated and undoubtedly malignant areas. These are usually epithelial or epithelial and mesenchymal (embryonal carcinoma and teratoma in the WHO classification ${ }^{11}$ ). In malignant teratoma undifferentiated (MTU) there is an absence of differentiated structure, the appearances ranging from an adenocarcinoma-like pattern to closely packed masses of cells which usually have a carcinomatous rather than sarcomatous appearance ${ }^{10}$ (embryonal carcinoma in the WHO classification ${ }^{11}$ ). The seminoma is a malignant tumour composed of solid masses of more or less uniform cells considered to have arisen from the germinal epithelium of the testis. Teratoma and seminoma of the testis may occur as separate pure tumours or together in a combined germ cell tumour. Their histological patterns are usually quite distinct but occasionally problems arise in distinguishing MTU from seminoma. ${ }^{12}$

\section{Material and methods}

\section{PRIMARY TUMOURS OF THE TESTES: BASELINE} MATERIAL

Smears were made from imprints or scalpel scrapes from the surfaces of 15 bisected fresh unfixed testicular tumours (seven seminoma, seven teratoma, one lymphoma). Of the seven teratoma, five were MTI, one MTU, and one yolk sac tumour. These were fixed immediately in $95 \%$ ethyl alcohol and stained using the Papanicalaou stain technique. Air dried smears were stained with May-Grünwald stain. In addition to the above group, smears were taken from three retroperitoneal masses resected after chemotherapy.
Table 1 Clinical material: primary tumours and sites of metastases

\begin{tabular}{lll}
\hline Site of primary & Histopathology & Site of metastases \\
\hline Left testis $(n=14)$ & Seminoma $(n=8)$ & $\begin{array}{l}\text { Para-aortic/ } \\
\text { intra-abdominal } \\
(n=18)\end{array}$ \\
$\begin{array}{lll}\text { Right testis }(n=9) \\
\text { Unrecorded }(n=4)\end{array}$ & $\begin{array}{l}\text { Teratoma }(n=13) \\
\text { MTD }(n=1)\end{array}$ & $\begin{array}{l}\text { Cung }(n=3) \\
\text { Cervical node }(n=3) \\
\text { CTI }(n=6)\end{array}$ \\
& MTU $(n=6)$ & \\
& Mixed teratoma/ $(n=1)$ \\
seminoma $(n=4)$ & Inguinal node $(n=1)$ \\
& Unknown $(n=2)$ & Scrotal wound $(n=1)$ \\
\hline
\end{tabular}

CLINICAL MATERIAL

The 27 patients in the series were aged between 29 and $67 ; 17$ were in the $20-40$ year age group. Table 1 shows the pathology of the primary tumours and sites of metastases.

The patients were divided into two groups: (A) Those with present or past evidence of a primary testicular tumour with metastases requiring urgent diagnosis and chemotherapy $(n=19)$; (B) those who had received one or more courses of chemotherapy for metastases from testicular tumour and were left with a residual mass or who developed a new mass under observation. There were 13 patients in this group including patients from group A whose initial diagnosis had been made by fine needle aspiration cytology.

Fine needle aspiration cytology was performed on 32 occasions using a 23 gauge needle from sites indicated in table 2 . Palpable masses were aspirated by direct puncture. Intrathoracic and intra-abdominal masses were aspirated by the radiologist on nodes opacified by lymphangiography or visualised by computed tomography. Smears were immediately fixed in 95\% ethyl alcohol and stained using the Papanicalaou technique. Air dried smears were stained with May-Grünwald stains.

\section{Results}

CYTOLOGICAL FEATURES OF PRIMARY

TESTICULAR TUMOURS: BASELINE MATERIAL

Smears from the resected seven primary classical

Table 2 Fine needle aspiration cytology diagnosis: metastasis from testicular tumours

\begin{tabular}{|c|c|c|c|c|}
\hline \multirow[b]{2}{*}{ Site } & \multicolumn{3}{|l|}{ Positive (malignant) } & \multirow{2}{*}{$\frac{\text { Negative }}{\text { (No viable tumour seen) }}$} \\
\hline & Malignant teratoma & Seminoma & Differentiated teratoma & \\
\hline $\begin{array}{l}\text { Intra-abdominal para-aortic } \\
\quad(n=22) \\
\text { Supraclavicular }(n=4) \\
\text { Lung }(n=3) \\
\text { Chest wall }(n=1) \\
\text { Inguinal node }(n=1) \\
\text { Orchidectomy scar }(n=1)\end{array}$ & $\begin{array}{l}6 \\
2 \\
2\end{array}$ & $\begin{array}{l}2 \\
1 \\
1 \\
1\end{array}$ & 1 & $\begin{array}{r}13 \\
1 \\
1\end{array}$ \\
\hline
\end{tabular}


seminomas of the testis showed the following features (figs 1-3). (i) Tumour cells were arranged in loose flat sheets and strands and singly with long cytoplasmic processes often running between groups of cells. (ii) Cytoplasmic margins were well defined and cytoplasm often showed a "glassy" quality. (iii) Nuclei were rounded or roughly polygonal and relatively uniform in shape and size. The chromatin was relatively fine and evenly distributed. There were one or more small or large nucleoli. Associated lymphocytes and groups of histiocytes varied from a few to numerous.

The cytological features in the smears from the seven testicular teratomas were determined by the pattern of differentiation of the tumour. The malignant teratomas with areas of papillary carcinoma (figs 4 and 5) showed the following features: (i) Tumour cells were arranged in tightly cohesive sheets, papillary clusters, or spherical masses. (ii) The cytoplasmic borders were relatively poorly defined. (iii) There were large, often irregular nuclei, varying in size and shape. (iv) The chromatin was fine or coarse and unevenly distributed. (v) One or more nucleoli were prominent. Lymphocytes were present in variable numbers.

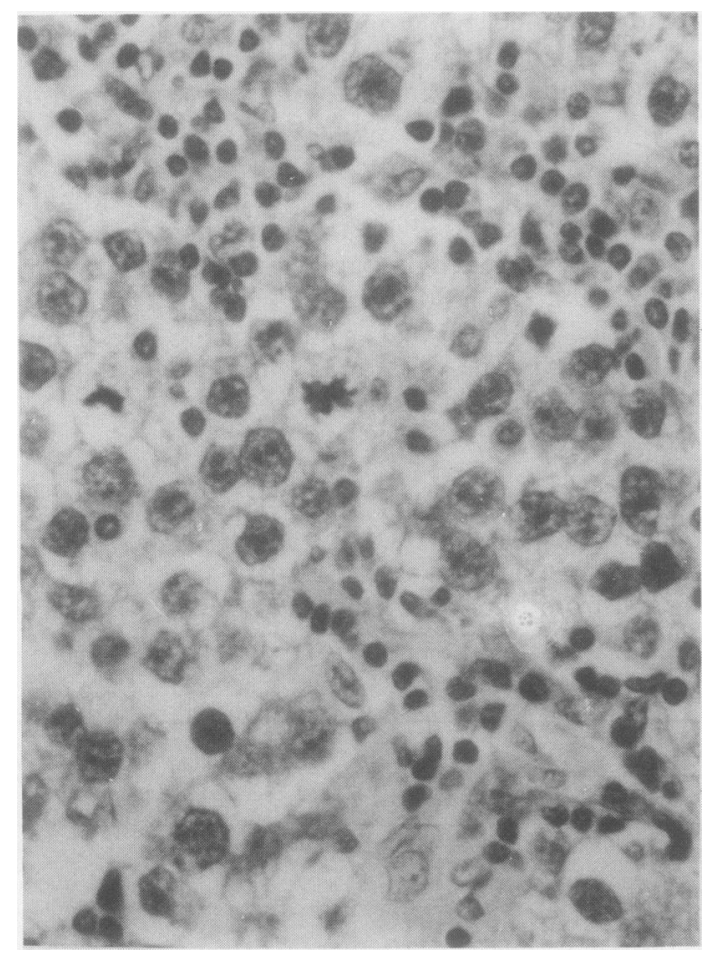

Fig 1 Seminoma of testis: histological section. (Haematoxylin and eosin.)

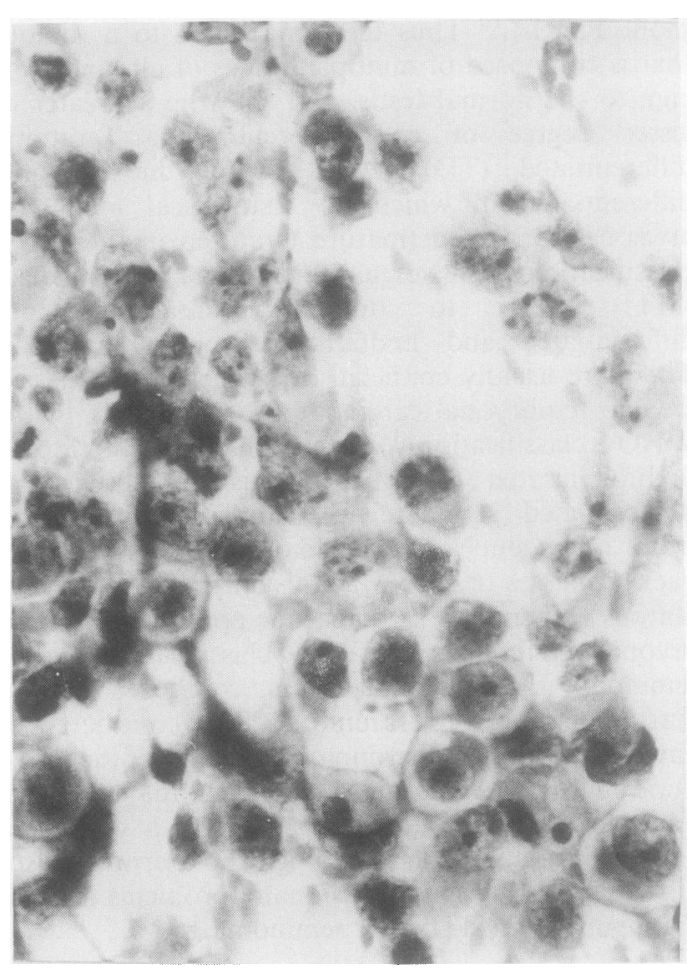

Fig 2 Seminoma of testis: cytological preparation. (Papanicalaou.)

Areas of solid undifferentiated carcinoma in the malignant teratomas appeared in the cytological preparations as syncitial sheets of cells wth nuclei considerably larger than seminomas and with pronounced nuclear pleomorphism (figs 6 and 7). The yolk sac tumour contained little which could be recognised as a separate entity in cytological preparations (fig 8).

Smears from three retroperitoneal masses resected after chemotherapy contained large sheets of benign columnar epithelium together with necrotic debris, histiocytes, and foreign body giant cells (fig 9). Histological sections showed the giant cells to be associated with necrotic debris and ruptured cysts lined by columnar or squamous epithelium in areas of differentiated teratoma.

The smears from the one case of primary immunoblastic lymphoma of the testis consisted of cells arranged both loosely and in closely packed groups. Nuclei were considerably smaller than in the germ cell tumours and were uniformly round with only occasional indentation. Chromatin was compact and there were one or more nucleoli. There was virtually no cytoplasm. There were no associated cells to suggest an inflammatory process (fig 10). 
A study of the cytological features of germ cell tumours in smears from fresh tissue enabled the following conclusions to be drawn. It would, on the whole, be possible to distinguish seminomas from teratomas in fine needle aspiration cytology material. The most striking difference lay in the arrangement of cells, which, in the teratomas, formed relatively tight cohesive sheets and papillary clusters with clear areas between; in seminomas, cells were spread across the field in loose flat sheets and strands, often associated with long cytoplasmic processed stretching between cells. The presence of clearly defined cell borders and glassy cytoplasm in seminomas was another helpful feature. Lymphocytes were not helpful in differential diagnosis as they were present in both seminoma and teratoma. The association of groups of epithelioid cells, especially when numerous, was of value in diagnosing seminoma. Differentiated teratomas could be recognised in smears from the presence of large sheets of benign columnar epithelium, often associated with histiocytes and large foreign body giant cells. When these sheets of benign columnar epithelium were associated with areas of clearly malignant epithelium MTI could be diagnosed cytologically.

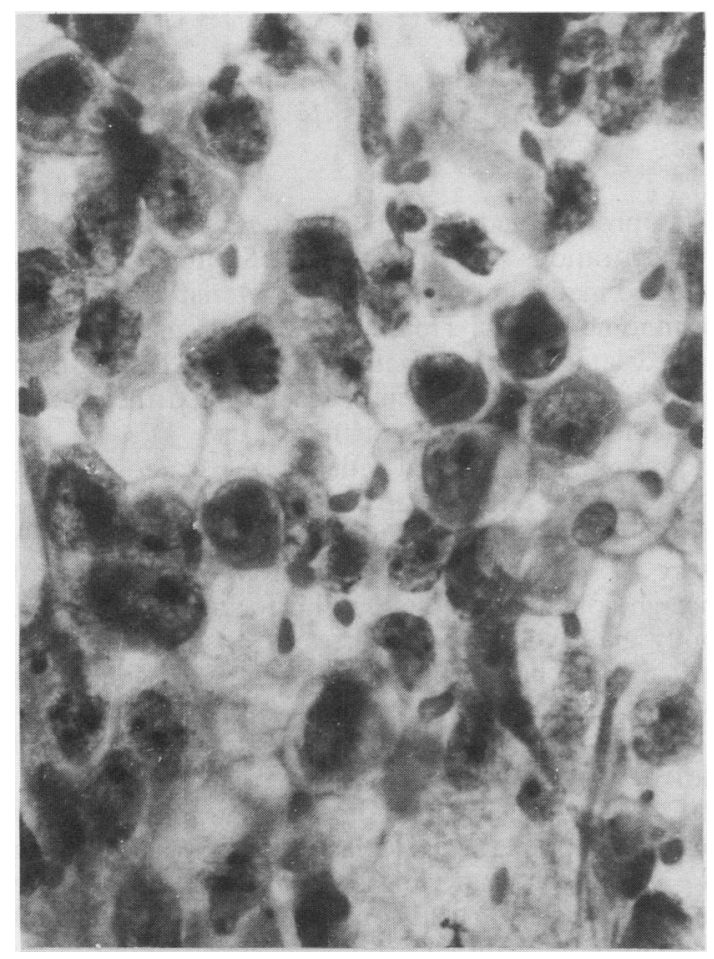

Fig 3 Seminoma of testis: cytological preparation. (Papanicalaou.)

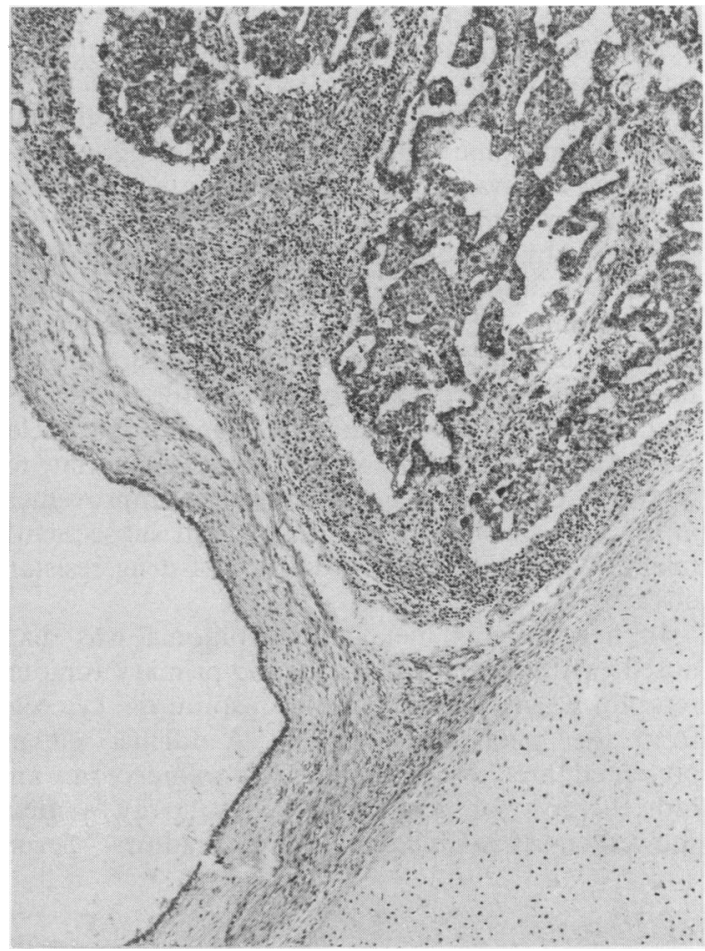

Fig 4 Malignant teratoma of testis, areas of differentiation coexist with papillary tumour (MTI): histological section. (Haematoxylin and eosin.)

\section{CYTOLOGICAL FINDINGS IN METASTASES} (table 2)

A positive diagnosis of viable metastatic tumour was made in 16 of the 32 aspirates. In 11 malignant teratoma was diagnosed; in two of these MTI was diagnosed based on the presence of groups of benign columnar epithelial cells in association with closely cohesive groups of malignant cells. In nine, malignant cells were present without any associated benign epithelium, five of which consisted of sheets of undifferentiated malignant cells and four consisted of papillary adenocarcinoma. One of the latter was an aspirate of a supraclavicular node from a patient whose primary testicular tumour was diagnosed as seminoma, with the suggestion of a papillary pattern at one edge of the section and a possible focus of MTU in a vein. This diagnosis was made on referred slides and no more material from the primary tumour was available for further examination. The fine needle aspiration cytology of the metastases was largely composed of papillary and undifferentiated carcinoma, indicating metastatic teratoma associated with relatively few cells that showed features charac- 
teristic of seminoma (fig 11). In an aspirate of lung deposits from a patient whose testicular tumour was a typical seminoma with no teratomatous elements, syncitial arrangement of the malignant cells with large pleomorphic and irregular nuclei suggested metastases composed of undifferentiated teratoma rather than seminoma (fig 12). This observation was supported by no noticeable change in the lung metastases on computed tomography after two courses of CIS platinum. The repeat fine needle aspiration cytology showed morphologically viable tumour, virtually unchanged by chemotherapy, except for an increase in the number of grossly enlarged pleomorphic nuclei. A change in the chemotherapy to that appropriate for teratoma produced a more impressive improvement in the lung metastases but this patient subsequently relapsed and died 18 months later of drug resistant disease.

In five patients metastatic seminoma was diagnosed, with all the features of the primary tumours reproduced in the fine needle aspiration cytology from the metastases (fig 13). A further patient presented three years later after left orchidectomy and radiotherapy for seminoma. Chest $x$-ray pictures showed massive bilateral hilar shadows. Serum



Fig 5 Malignant teratoma of testis. Papillary cluster of malignant cells: cytological preparation. (Papanicalaou.)

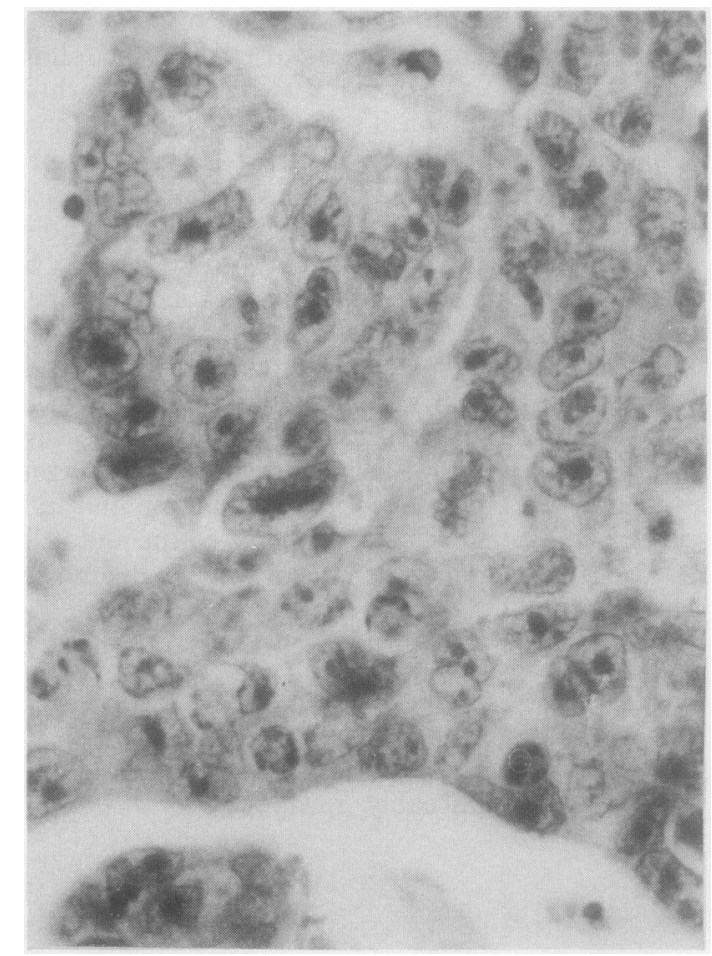

Fig 6 Malignant teratoma of testis. Area of undifferentiated carcinoma: histological section. (Haematoxylin and eosin.)

$\alpha$ fetoprotein and human chorionic gonadotrophin concentrations were normal. Sputum cytology showed malignant cells with characteristic features of seminoma (fig 14). Treatment with CIS platinum followed later by bleomycin, etoposide, CIS platinum (BEP) produced rapid improvement and the patient remained well at three year follow up.

One patient presented with a large para-aortic mass 11 years after radiotherapy for MTI. Fine needle aspiration cytology showed that the cytological features were those of differentiated teratoma with sheets of benign columnar epithelium, histiocytes, and foreign body giant cells. Two courses of chemotherapy resulted in no noticeable reduction in size of the mass which was excised and found to consist of fibrous tissue and mucus lined cysts, representing residual differentiated teratoma.

Of the 15 negative fine needle aspiration cytology results, 14 preparations consisted only of blood with no diagnostic material and one contained large masses of necrotic cells together with histiocytes and foreign body giant cells but no viable tumour. This group included 10 patients with residual masses after completion of chemotherapy. 


\section{ACCURACY OF FINE NEEDLE ASPIRATION CYTOLOGY}

The accuracy of fine needle aspiration cytology was evaluated from follow up histology, clinical assessment, serum markers, radiological examinations, in particular, computed tomography and ultrasound examinations, and response to chemotherapy assessed by dissappearance of noticeable reduction in size of metastases and return of serum markers to normal.

Histological examination after aspiration was done in eight patients, all of whom had received previous chemotherapy or radiotherapy for metastatic disease. Seven were resected masses of retroperitoneal lymph nodes and one a nodule in the chest wall. In this group three positive cytology reports of TD, MTU, and seminoma were confirmed histologically. In the remaining five with negative fine needle aspiration cytology (blood alone in four, necrotic cells in seven), the histology showed TD in three and completely necrotic tumour in two.

The serum markers analysed were $\alpha$ fetoprotein, human chorionic gonadotrophin, hydroxy butyric dehydrogenase and in some cases placental alkaline phosphatase. Noticeably raised concentrations of



Fig 7 Malignant teratoma of testis. Sheet of poorly differentiated malignant cells: cytological preparation. (Haematoxylin and eosin.)



Fig 8 Yolk sac tumour of testis: histological section. (Haematoxylin and eosin.)

serum human chorionic gonadotrophin were recorded in seven of the 13 patients with metastases from teratomas of the testes. The patient whose primary testicular tumour was seminoma with the suggestion of associated teratoma had high concentrations of serum human chorionic gonadotrophin. This supported the findings from fine needle aspiration cytology of the supraclavicular node metastases which were almost entirely teratoma apart from a few areas of seminoma cells. Serum placental alkaline phosphatase was raised in one patient with seminoma but was not recorded in every case.

In all cases in which urgent chemotherapy was undertaken on the basis of positive cytology supported by clinical, radiological, and serum marker indication of active disease, a rapid response provided further evidence of reliable cytological diagnosis. All patients remained well on follow up of between six months and four and a half years, with two exceptions. One patient with advanced MTU had been treated elsewhere after laparotomy and tissue excision to confirm the diagnosis. Chemotherapy started three weeks later was followed by severe ileus, leucopenic sepsis, and oliguric renal failure. Severe postoperative morbidity followed chemotherapy. This, together with ex- 
tensive disease, resulted in death after eight months. The other was the patient referred to in the previous paragraph.

\section{Discussion}

This study aimed to define the morphological features of germ cell tumours of the testis in cytological preparations made from fresh resected tumours and apply them to the fine needle aspiration cytology diagnosis of metastases. Delay in diagnosis is an adverse prognostic factor affecting both the risk of growth of metastases and the chance of cure by cytotoxic drugs. ${ }^{2}$ Laparotomy to obtain tissue for diagnosis is undesirable in patients whose capacity for postoperative recovery is undermined by serious disease and in whom a surgical complication may cause considerable delay in starting treatment. Intensive chemotherapy in the postoperative period may hinder the healing process and contribute to complications, as occurred in one patient in this series. In interpreting the fine needle aspiration cytology material the cytologist has the following responsibilities: (i) to ascertain whether viable malignant tumour is present or absent; (ii) when tumour is present in the fine needle aspi-



Fig 9 Retroperitoneal mass. Teratoma differentiated: cytological preparation. (Papanicalaou.)

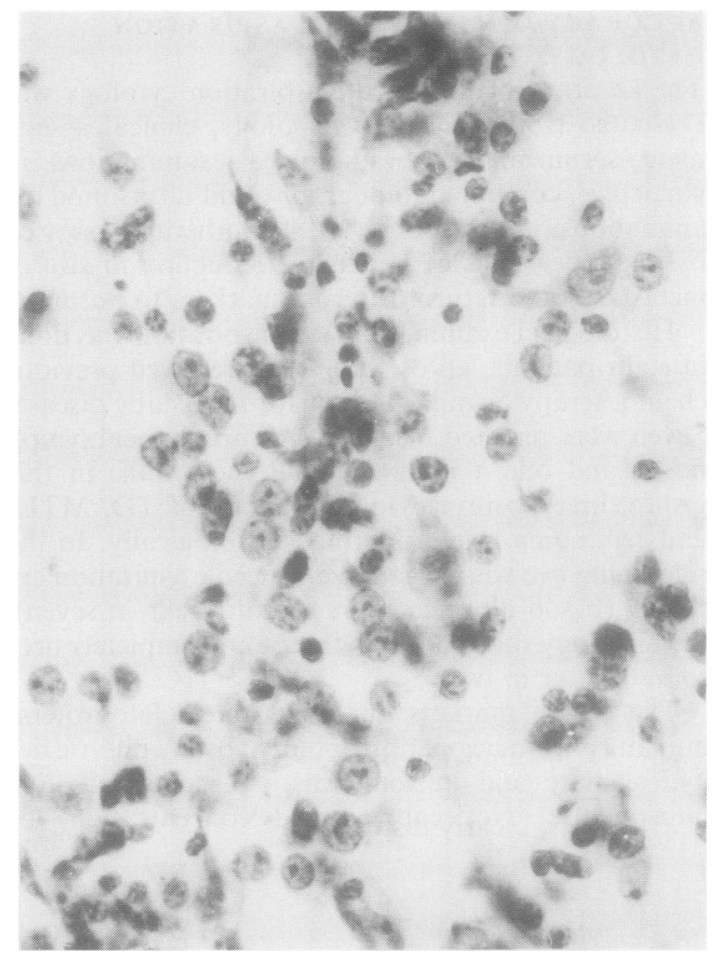

Fig 10 Primary lymphoma of testis: cytological preparation. (Papanicalaou.)

ration cytology material, to establish that this represents spread from a testicular tumour and not a second primary; (iii) where possible to indicate the testicular tumour type.

As a positive report of viable testicular tumour metastasis necessitates treatment with powerful chemotherapeutic agents with their attendant unpleasant side effects, a false positive report is highly undesirable. On the other hand, a reliable positive fine needle aspiration cytology report of tumour supported by clinical, radiological, and serum marker evidence of active disease allows urgent chemotherapy to be started without laparotomy and tissue diagnosis, thereby saving both time and postoperative morbidity. When used after chemotherapy, fine needle aspiration cytology may also save patients with residual malignancy from surgical staging. ${ }^{1}$ The likelihood of impotence following resection of large residual para-aortic masses rendered this an unacceptable means of excluding persistent tumour in several patients in the series. As with all cytology, as negative report does not exclude the presence of tumour. Sampling of metastases from testicular tumours is a major problem because of the considerable size of the metastases, the range of tissue represented, 
and the extensive necrosis which follows chemotherapy. One $6 \mathrm{~cm}$ mass resected after chemotherapy was almost entirely necrotic except for two $0.3 \mathrm{~cm}$ foci of seminoma found after examining 11 sections. The chances of sampling such a focus by fine needle aspiration cytology must be minimal and that of a false negative report high. Sampling of both primary and metastatic tumour can be a problem for the histopathologist, requiring numerous sections to be examined.

Treatment of metastatic germ cell tumours depends not only on stage of tumour but also histological type. The catagorisation of the different types of testicular tumour in the cytological preparations proved a reliable exercise on the whole. The cytological features shown in smears from the seminomas were sufficiently distinctive for an unequivocal diagnosis to be made on cytological material alone. Differentiated teratoma was diagnosable when large sheets of benign columnar epithelium were found associated with histiocytes and foreign body giant cells. When this material was found in association with malignant tissue, MTI could be diagnosed. Metastases from MTU could be clearly distinguished from seminoma

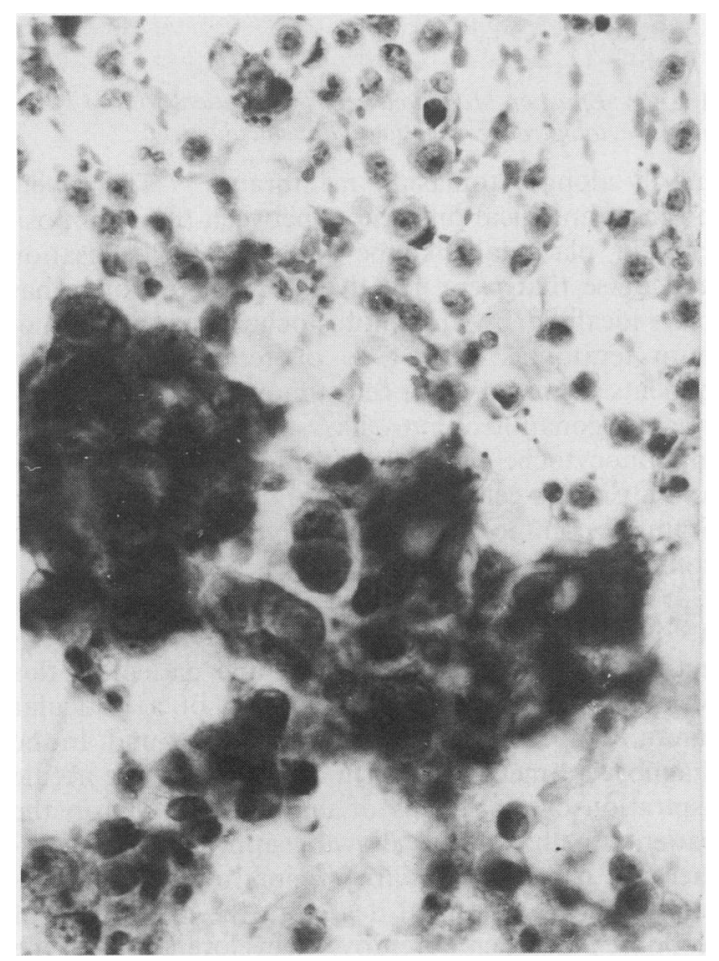

Fig 11 Fine needle aspiration cytology of supraclavicular node. MTU and seminoma: cytological preparation. (Papanicalaou.)

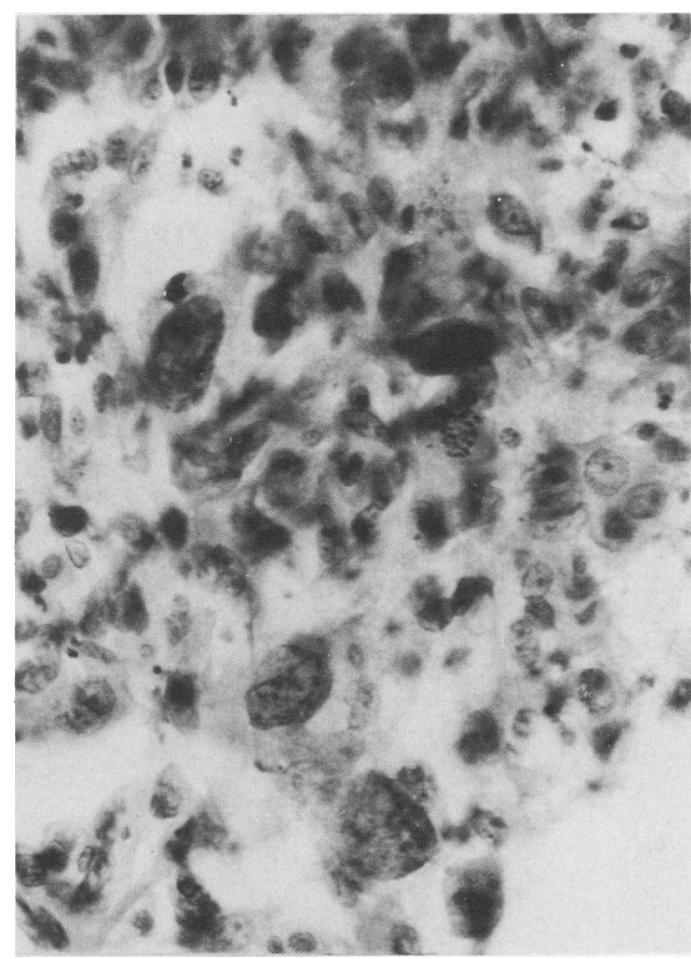

Fig 12 Fine needle aspiration cytology of lung. Undifferentiated teratoma: cytological preparation. (Papanicalaou.)

and a metastasis from combined MTU and seminoma showed the features of both. It would, however, not always be possible to distinguish MTU or MTI metastases from papillary or undifferentiated carcinoma from another primary site. Cells with large pleomorphic nuclei containing multiple nucleoli, bizarre giant cells, and multinucleate syncitial forms would be helpful in diagnosing MTU. Comparison of the features in fine needle aspiration cytology material with the histology of the primary tumour should be of help when available. The value of conventional cytology for diagnosis of metastatic testicular tumour should not be forgotten. Metastatic seminoma can be clearly recognised in sputum from patients with lung metastases. Effusions may contain cells with recognisable features of metastatic testicular tumour (fig 15).

Immunohistological stains for tumour markers were not used in this study beca7use urgent and rapid diagnosis based on readily recognised morphological criteria was required. Immunohistochemical markers, although of undoubted value, are not necessarily helpful in every case. Uchida et al have shown placental alkaline phosphatase in $90 \%$ of seminoma, pri- 


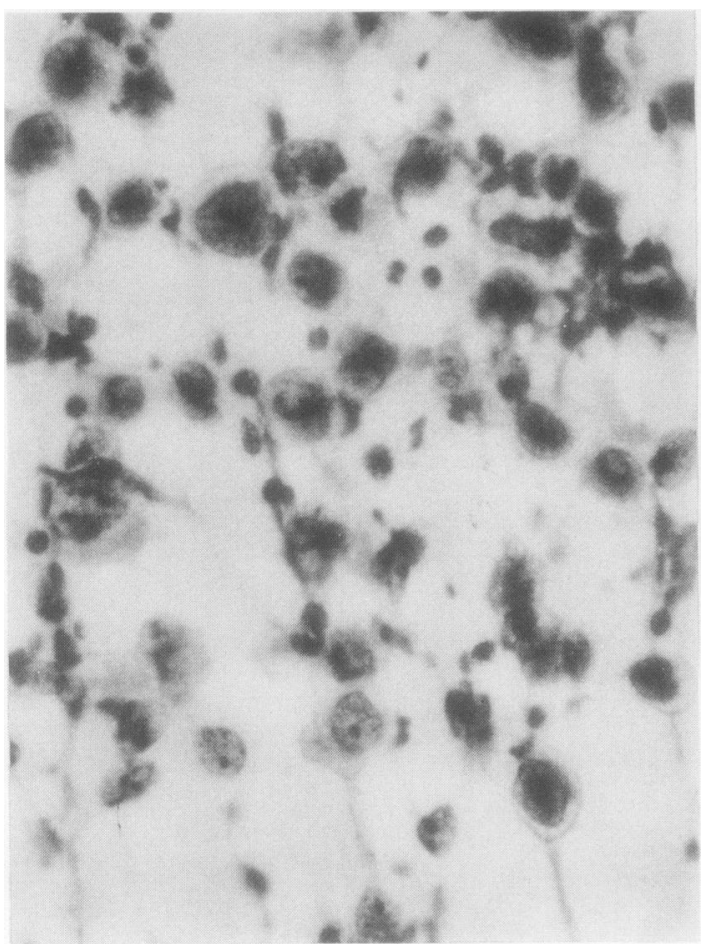

Fig 13 Fine needle aspiration cytology of para-aortic lymph node. Seminoma: cytological preparation. (Papanicalaou.)

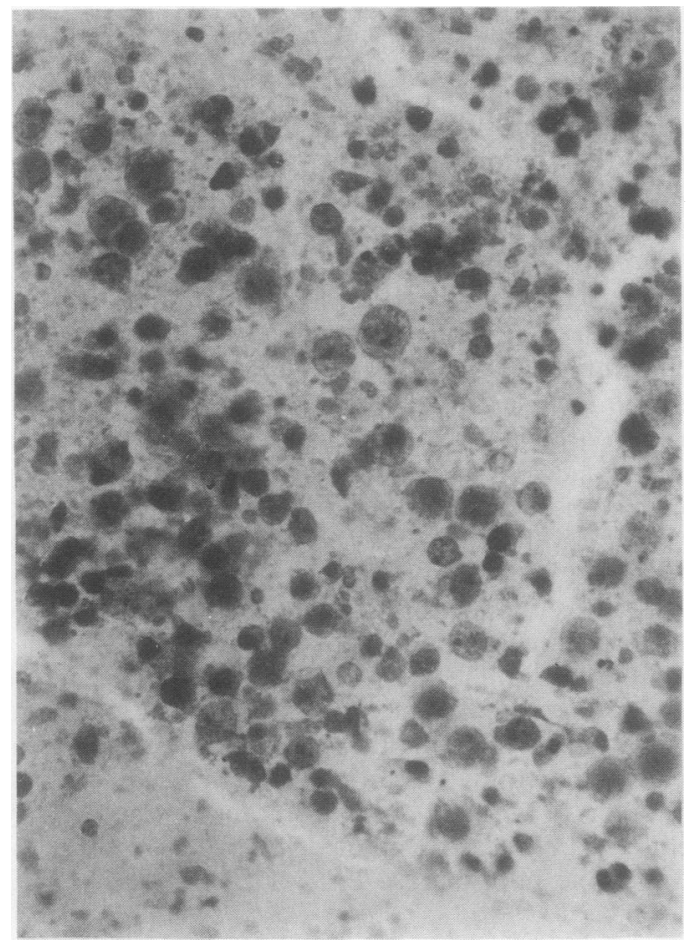

Fig 14 Sputum: seminoma cells: cytological preparation. (Papanicalaou.)

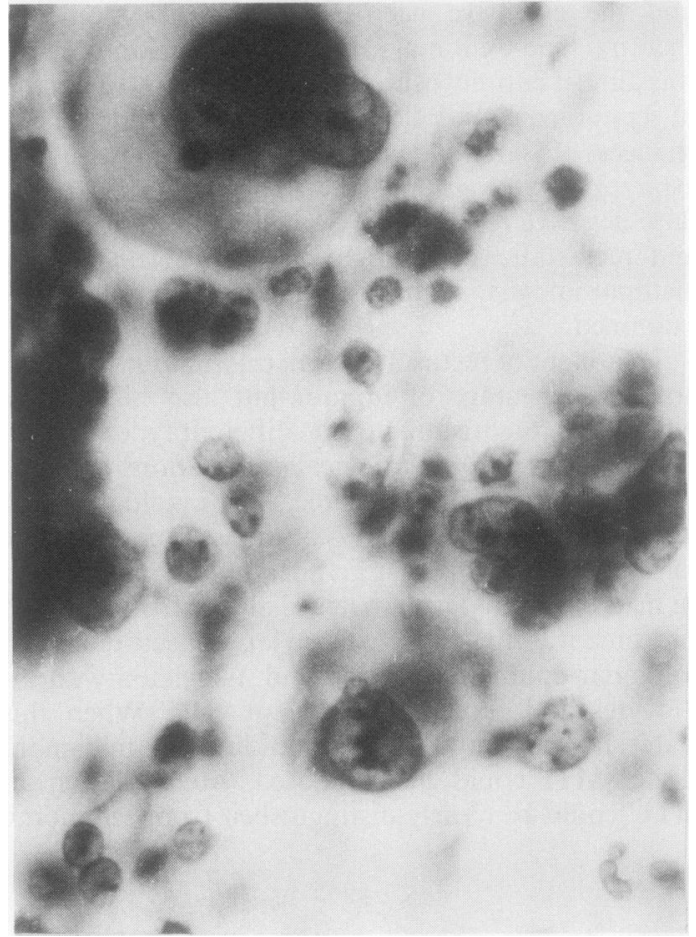

Fig 15 Effusion. Malignant cells from patient with MTU of testis: cytological preparation. (Papanicalaou.)

marily along cytoplasmic membranes. ${ }^{13}$ There were no morphological differences between tumours positive for placental alkaline phosphatase localisation and those that were negative. Alpha fetoprotein has been localised by immunohistochemistry in $20 \%$ of pure teratomas and $47 \%$ of teratomatous components of mixed germ cell tumours. ${ }^{13}$ Human chorionic gonadotrophin has been localised by immunocytochemistry in trophoblastic teratomas and in similar cells found in $2-14 \%$ of seminomas. ${ }^{13}$ Stain for common leucocyte antigen could be used to confirm a diagnosis of lymphoma.

It must be emphasised that we do not advocate fine needle aspiration cytology for the diagnosis of the primary testicular tumour. Indeed, one patient in this series referred after scrotal excision of a testicular tumour had a metastasis in the scrotal wound. In the diagnosis of metastatic germ cell tumours fine needle aspiration cytology was of undoubted value in the patients in this series, allowing appropriate chemotherapy to be started with minimal delay and morbidity, and to a limited degree in the assessment of response to chemotherapy. Development of the radioimmunodetection technique with reliable markers can be expected to improve the accuracy of sampling and enhance the value of diagnosis by fine needle aspiration cytology. ${ }^{14}$

We thank Drs Constance Parkinson and Juliet Heaton for the cytological preparations of "baseline 
material" obtained from the fresh surgical specimens and for access to histological sections and reports, and Dr MJ Kellett for the fine needle aspiration cytology material.

\section{References}

1 Vogelzang NJ. Clinical management of patients with testicular tumours. In: Talerman A, Roth LM, eds. Pathology of the testis and its adnexae. New York: Churchill Livingstone, 1986:207-19.

2 Oliver RTD, Highman WJ, Kellett MJ, Curling OM, Dacie JE. The value of fine needle aspiration cytology in the management of metastatic germ cell tumours. Br J Urol 1985;57:200-3.

3 Zajicek J. Testes and epdidymis. In: Aspiration biopsy cytology. Part 2: cytology of infradiaphragmatic organs. London: S Karger, 1979:104-28.

4 Droese M, Rahlf G. Punktionszytologie Maligner Hodentumoreu? Urologe 1979;A18:338-42.

5 Ecklund L, Gothlin J. Fine needle biopsy of metastases at retrograde pyelography. Scand J Urol Nephrol 1976;10:261-2.

6 Zornoza J, Handell P, Lukeman JM, Jing BS, Wallace S. Percutaneous transperitoneal biopsy in urological malignancy. Urology 1977;IX:395-8.

7 Wajsman Z, Gamarra M, Park J, Beckley S, Pontes JE. Transabdominal fine needle aspiration of retroperitoneal lymph nodes in staging of genitourinary cancer. $J$ Urol 1982;
128:1238-40.

8 Wajsman Z, Gamarra M, Park J, Beckley S, Pontes JE, Murphy $\mathrm{G}$. Fine needle aspiration of metastatic lesions and regional lymph nodes in genitourinary cancer. Urology 1982;IX:356-60.

9 Pugh RCB. Testicular tumours. In: Pugh RCB, ed. Pathology of the testes. Oxford: Blackwell Scientific Publications, 1976: 139-59.

10 Pugh RCB, Cameron K. Teratoma. In: Pugh RCB, ed. Pathology of the testes. Oxford: Blackwell Scientific Publications, 1976:199-244.

11 Mostofi FK, Sobin LH. Histological typing of testicular tumours. In: International histological classification of tumours No 16. Geneva: World Health Organisation, 1977:27-31.

12 Alderdice JM, Johnson SR. Embryonal carcinoma of testis simulating seminoma. J Clin Pathol 1987;40:434-7.

13 Klappenbach RS, Kurman R. Immunohistochemistry of germ cell tumours. In: Talerman A, Roth LM, eds. Pathology of the testis and its adnexae. New York: Churchill Livingstone. 1986:169-79.

14 Javadpour N. Value of tumour markers in testicular cancer. In: Talerman A, Roth LM, eds. Pathology of the testis and its adnexae. New York: Churchill Livingstone, 1986:221-30.

Requests for reprints to: Dr Wilma J Highman, Department of Cytology, Middlesex Hospital, Mortimer Street, London WC1. 